\title{
Erratum to: Use of EOS imaging for the assessment of scoliosis deformities: application to postoperative 3D quantitative analysis of the trunk
}

Brice Ilharreborde · Jean Dubousset • Jean-Charles Le Huec

Published online: 23 July 2014

(c) Springer-Verlag Berlin Heidelberg 2014

Erratum to: Eur Spine J

DOI 10.1007/s00586-014-3334-7

The author line should read:

Brice Ilharreborde, Jean Dubousset, Jean-Charles Le Huec.

The online version of the original article can be found under doi:10.1007/s00586-014-3334-7.

B. Ilharreborde

Pediatric Orthopaedic Department, Robert Debré Hospital, Paris,

France

J. Dubousset ( $\bowtie)$

Académie Nationale de Médecine, 23 bis rue des cordelières,

75013 Paris, France

e-mail: jean.dubousset@wanadoo.fr

J.-C. Le Huec

Spine Unit, Orthopaedic Department, CHU Bordeaux, Bordeaux,

France 